CASE REPORT

\title{
Multiple microcarcinoids in a patient with long standing ulcerative colitis
}

\author{
T Matsumoto, Y Jo, R Mibu, M Hirahashi, T Yao, M lida
}

J Clin Pathol 2003;56:963-965

A male patient with a 17 year history of intractable ulcerative colitis of the entire type was treated by total proctocolectomy. Colonoscopy before surgery did not identify dysplasia. Histological examination of the resected colorectum revealed that, in addition to chronic inflammatory infiltrates, there were 21 areas of microcarcinoids located in the muscularis propria and in the superficial layer of the submucosa. Carcinoids may be more common than previously thought, and they may be a reactive phenomenon to a variety of factors in ulcerative colitis.

$\mathrm{t}$ is well known that patients with long standing ulcerative colitis (UC) are prone to the development of colorectal neoplasia. ${ }^{1-3}$ Among various types of colorectal tumour, carcinoma has an extremely high frequency, followed by malignant lymphoma. There have also been reported cases of colorectal carcinoids complicating UC. ${ }^{4}$ However, the association between inflammatory bowel disease (IBD) and carcinoid tumours has been questioned, because the incidence of carcinoid tumours in patients with IBD is thought to be similar to that seen in subjects without IBD, and tumours occur predominantly in parts of the intestine not affected by IBD. ${ }^{5}$

\section{"Among various types of colorectal tumour, carcinoma has an extremely high frequency, followed by malignant lymphoma"}

Here, we report a patient with UC in whom microcarcinoids were incidentally found in the resected colorectum.

\section{CASE REPORT}

A Japanese man was initially diagnosed with UC of the entire type in August 1984, when he was 35 years of age. Since then, he has been treated by oral prednisolone and mesalazine. Even though these medications were transiently effective, the disease frequently relapsed and the patient required repeated hospitalisation. After the fourth admission in August 2000, the patient was treated with oral predonisilone $(30 \mathrm{mg} /$ day) and $2.0 \mathrm{~g}$ of mesalazine. However, the disease relapsed in August 2001.

Because of the intractable clinical course, we decided that the patient should undergo total proctocolectomy. Before surgery, he was treated by granulocyte apheresis, which improved his symptoms. At that time, we performed colonoscopy with multiple biopsies. Colonoscopically, the affected colorectum manifested coarse and friable granular mucosa, extending from the rectum to the hepatic flexure, and multiple small ulcers in the sigmoid colon. However, no dysplasia associated lesions or masses were seen. Four biopsy specimens were obtained from the colon, in which dense inflammatory infiltrates without epithelial dysplasia were detected. The patient was subsequently treated by total proctocolectomy and ileoanal anastomosis.

Macroscopic examination of the resected specimen revealed that the colon was thickened with fine granular mucosa (fig 1A). There were no protruding or depressed lesions. Microscopically, chronic inflammatory infiltrates and degenerated glands were seen throughout the colon and the rectum. Furthermore, there were multiple small islands of large cells in the muscularis mucosae and in the upper layer of the submucosa in the distal sigmoid colon and in the rectum (fig 2). These cells were arranged in a trabecular fashion, and they were characterised by pale, eosinophilic cytoplasm and ovoid nuclei. In addition, the cells had argyrophilic granules and were positive for Grimelius and chromogranin stains. These histological features were compatible with microcarcinoids. As shown in fig 1B, thorough sectioning of the resected specimen revealed that there were 21 areas of microcarcinoid in the resected colorectum.

\section{DISCUSSION}

Carcinoid tumours are neoplasms that arise from mature endocrine cells or their stem cells within the epithelial layer of the gastrointestinal tract. In 1979, Hay and Curt ${ }^{6}$ reported a case of carcinoid tumour complicating UC. Since then, 21 cases of UC complicated by histologically verified carcinoid tumour have been described in the English literature. ${ }^{4-16}$ As has been the case in our patient, almost all carcinoids were diagnosed histologically in the surgically removed specimens. Although the incidence of carcinoid in UC remains obscure, our review of the literature suggests that the occurrence of carcinoid is an exceptional event in patients with UC. In addition, it should be noted that there is a considerable heterogeneity in the histology and the site of carcinoids. ${ }^{4-16}$

There has been controversy as to the association of carcinoid and UC. Greenstein et al reported five cases of UC accompanied by carcinoids. ${ }^{5}$ However, their patients had carcinoids at sites apparently unaffected by UC (the appendix and the ileum), where the tumour occurs predominantly in the general population. ${ }^{17}{ }^{18}$ Thus, Greenstein et al suggested that the development of carcinoids is independent of UC. ${ }^{5}$ Another case of appendiceal carcinoid complicating UC was described by LeMarc'hadour et al. ${ }^{14}$ However, the development of carcinoid in the appendix in UC does not exclude the possible correlation of the two conditions, because recent investigations have confirmed that the appendix can be affected by UC. ${ }^{19}{ }^{20}$ Miller and Summer ${ }^{15}$ and Dodd ${ }^{16}$ suggested a possible contribution of chronic inflammation to the development of carcinoids, because they found

Abbreviations: IBD, inflammatory bowel disease; UC, ulcerative colitis 

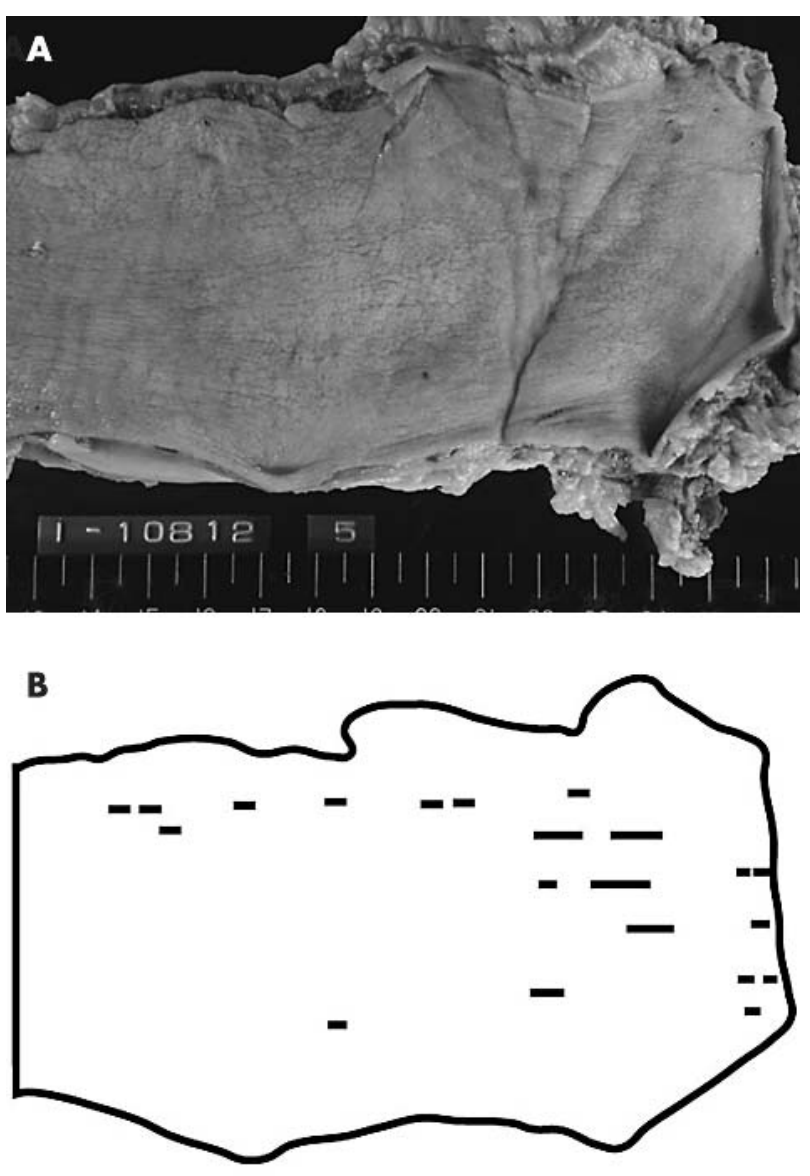

Microcarcinoid

Figure 1 Macroscopic findings of the resected colon. (A) The colorectal mucosa shows fine granularity. There are no protrusions or depressions. (B) A scheme to illustrate the positions of the microcarcinoids, which are indicated by the lines.

enteroendocrine cell hyperplasia in the flat mucosa of patients with UC complicated by solid carcinoid tumour.

"It is possible that microcarcinoids in UC are a reactive phenomenon induced by chronic inflammation"

Our case was characterised by a 17 year history of intractable UC and the development of multiple microcarcinoids. There have been two reported cases of UC accompanied microcarcinoids similar to those found in our patient. ${ }^{12}{ }^{13}$ In those earlier reports, microcarcinoids were found in the left side of the large bowel. In a patient of McNeely et al, ${ }^{12}$ multiple carcinoids were identified in a hemicolectomised specimen, which also contained invasive sigmoid colon cancers and dysplasia. In the other case, a solitary microcarcinoid was incidentally found in the rectum of a patient operated on because of intractable UC. ${ }^{13}$ It has been shown that enteroendocrine cell hyperplasia is a common histological feature in UC. ${ }^{14}$ Furthermore, epithelial stem cells, in addition to columnar cells, can be damaged by inflammation. Thus, it is possible that microcarcinoids in UC are a reactive phenomenon induced by chronic inflammation.

Even if carcinoids in UC can be presumed to be a consequence of chronic inflammation, it still remains unclear how and to what extent the inflammation contributes to the pathogenesis of carcinoids. However, it is possible that
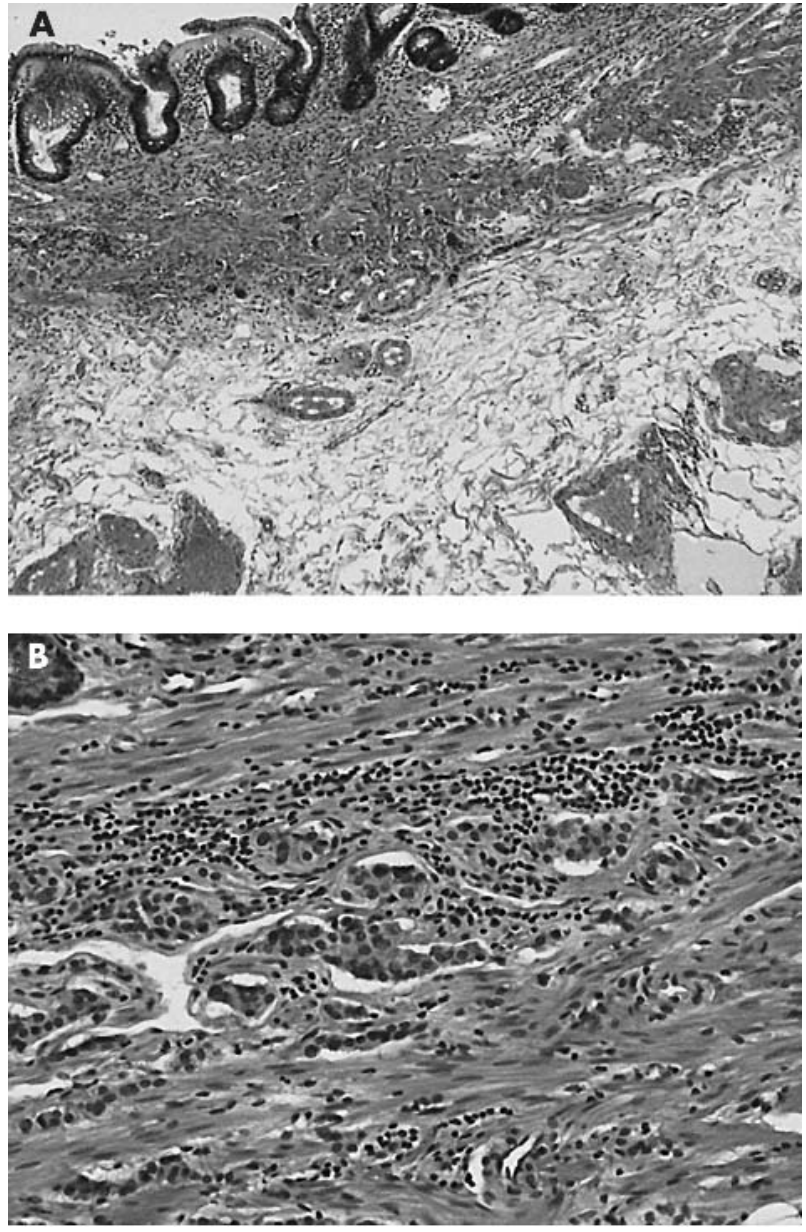

Figure 2 Histological findings of the resected sigmoid colon. (A) Low power magnification shows chronic inflammatory infiltrates in the lamina propria and in the submucosa. There are large cells with round nuclei in the muscularis mucosae and in the upper submucosal layer. (B) High power magnification reveals that the cells are arranged in a trabecular fashion and have round nuclei and pale cytoplasm. These features are compatible with carcinoid tumour.

microcarcinoids remain undetected in patients with UC, because they can easily be missed by colonoscopy, as in our patient. ${ }^{4}$ In addition, biopsy specimens are inappropriate for the diagnosis because of the predominant submucosal nature of the tumour. Whereas diagnostic and therapeutic strategies for dysplasia and cancer in UC have been rigorously investigated during the past 30 years, such strategies need to be investigated for carcinoid tumours.

\section{Take home messages}

- We report a patient with intractable ulcerative colitis of the entire type in whom multiple microcarcinoids were found in the resected colorectum after total proctocolectomy, although they were not noted on previous biopsies

- Carcinoids may be more common than previously thought, and they may be a reactive phenomenon to a variety of factors in ulcerative colitis

- Diagnostic and therapeutic strategies for carcinoid tumours need to be investigated 


\section{Authors' affiliations}

T Matsumoto, Y Jo, M lida, Department of Medicine and Clinical Science, Graduate School of Medical Sciences, Kyushu University, Maidashi 3-1-1, Higashi-ku, Fukuoka 812-8582, Japan

R Mibu, Clinical and Surgical Oncology, Graduate School of Medical Sciences, Kyushu University

M Hirahashi, T Yao, Anatomic Pathology, Graduate School of Medical Sciences, Kyushu University

Correspondence to: DrT Matsumoto, Department of Medicine and Clinical Science, Graduate School of Medical Sciences, Kyushu University, Maidashi 3-1-1, Higashi-ku, Fukuoka 812-8582, Japan; matane@intmed2.med.kyushu-u.ac.jp

Accepted for publication 11 June 2003

\section{REFERENCES}

1 Ekbom A, Helmick C, Zack M, et al. Ulcerative colitis and colorectal cancer: a population-based study. N Engl J Med 1990;323:1228-33.

2 Karlén $P$, Löfberg $R$, Broström $O$, et al. Increased risk of cancer in ulcerative colitis: a population-based cohort study. Am J Gastroenterol 1999;94:1047-52.

3 Connell WR, Talbot IC, Harpaz N, et al. Clinicopathological characteristics of colorectal carcinoma complicating ulcerative colitis. Gut 1994;35:1419-23.

4 Farr CM, Rajala WP. Carcinoid tumor complicating ulcerative colitis. Gastrointest Endosc 1990;36:315-16.

5 Greenstein AJ, Balasubramanian S, Harpaz N, et al. Carcinoid tumor and inflammatory bowel disease. A study of eleven cases and review of the literature. Am J Gastroenterol 1997;92:682-5.
6 Hay DJ, Curt JRN. Carcinoid tumor as a complication of ulcerative colitis. Postrad Med J 1979;55:430-2.

7 Owen DA, Hwang WS, Thorlakson RH, et al. Malignant carcinoid tumor complicating chronic ulcerative colitis. Am J Clin Pathol 1981;76:333-8.

8 Barki Y, Boult I. Two uncommon malignancies complicating chronic ulcerative colitis. J Can Assoc Radiol 1981;32:136-7.

9 Lyss AP, Thompson JJ, Glick JH. Adenocarcinoid tumor of the colon arising in preexisting ulcerative colitis. Cancer 1981;48:833-9.

10 Gledhill A, Hall PA, Cruze JP, et al. Enteroendocrine cell hyperplasia, carcinoid tumor and adenocarcinoma in longstanding ulcerative colitis. Histopathology 1986;10:501-8.

11 Sauven P, Ridge JA, Quan SH, et al. Anorectal carcinoid tumors: is aggressive therapy warranted? Ann Surg 1990;211:112-16.

12 McNeely B, Owen DA, Pezim M. Multiple microcarcinoids arising in chronic ulcerative colitis. Am J Clin Pathol 1992;98:112-16.

13 Haidar A, Dixon MF. Solitary microcarcinoid in ulcerative colitis. Histopathology 1992;21:487-8.

14 LeMarc'hadour F, Bost F, Peoc'h J, et al. Carcinoid tumour complicating inflammatory bowel disease. Pathol Res Pract 1994; 190: 1 185-92.

15 Miller R, Summer HW. Agyrophilic cell hyperplasia and an atypical carcinoid tumor in chronic ulcerative colitis. Cancer 1982;50:2920-5.

16 Dodd SM. Chronic ulcerative colitis complicated by atypical carcinoid tumor. $J$ Clin Pathol 1986;39:913-16.

17 Modlin IM, Sandor A. An analysis of 8305 cases of carcinoid tumors. Cancer 1997;79:813-29.

18 Hemminki K, Li X. Incidence trends and risk factors for carcinoid tumors Cancer 2001; 92:2204-10.

19 Scott IS, Sheaff M, Coumbe A, et al. Appendiceal inflammation in ulcerative colitis. Histopathology 1998;33:168-73.

20 Jo Y, Matsumoto T, Yada S, et al. Histological and immunological features of the appendix in patients with ulcerative colitis. Dig Dis Sci 2003;48:99-108. 